\title{
Fuzzy call admission control combined with distributed dynamic channel assignment and reassignment for cellular mobile systems
}

\author{
Shih-Cheng Horng ${ }^{1 *}$ and Shieh-Shing Lin ${ }^{2}$
}

\begin{abstract}
In a cellular mobile system (CMS), the service area is divided into cells, each of which has numerous channels, which are shared by two types of call - new calls and handoff calls. Giving a higher priority to handoff calls than new calls is common practice. However, giving too much priority to handoff calls will result to excessive blocking of new calls. This work firstly proposes a distributed dynamic channel assignment and reassignment (DDCAR) scheme to satisfy three types of constraint - co-channel constraint (CCC), adjacent channel constraint (ACC), and co-site constraint (CSC), simultaneously. The purpose is to minimize the number of available channels that become unavailable for the assignment to new calls and to maximize the number of unavailable channels that become available for release when calls complete. To provide a higher priority to handoff calls, a fuzzy call admission control (FCAC) scheme, combined with the DDCAR, is proposed herein for implementation at the base station. A $7 \times 7$ CMS with two traffic patterns is employed as the test example. The test results reveal that the FCAC scheme significantly reduces the dropping probability of handoff calls at the cost of increasing the blocking probability of new calls to an acceptable level.
\end{abstract}

Keywords: Call admission control; Cellular mobile system; Channel interference; Dynamic channel assignment; Fuzzy inference system; 7-cell reuse cluster system

\section{Introduction}

Nowadays, the cellular mobile system (CMS) has become one of the most profitable mobile systems because more applications are being made available for smart phones, which also have very attractive functions $[1,2]$. One of the most important issues in the management of a CMS concerns the effective utilization of the limited resources of the radio spectrum and the maintenance of communication quality. To address this issue, channel assignment is required. In a CMS, three types of interference are generally treated as constraints; these are the co-channel constraint (CCC), the adjacent channel constraint (ACC), and the co-site constraint (CSC) [3,4]. The CCC states that any two cells within the channel reuse distance cannot use the same channel. The ACC

\footnotetext{
* Correspondence: schong@cyut.edu.tw

'Department of Computer Science and Information Engineering, Chaoyang University of Technology, 168 Jifong E. Rd., Wufeng District, Taichung City 41349, Taiwan

Full list of author information is available at the end of the article
}

implies that adjacent channels cannot be assigned to adjacent cells. The CSC is that any pair of channels in the same cell must be a specified distance apart.

To maintain the quality of communication, channel assignment methods must incorporate the aforementioned constraints. The numerous channel assignment schemes include fixed channel assignment (FCA), dynamic channel assignment (DCA), borrowing channel assignment (BCA), and hybrid channel assignment (HCA) [5-9]. Among these, DCA schemes [8,9] are considered to be the most flexible and effective for improving the blocking probability. These schemes are divided into two categories - centralized schemes and distributed schemes [9]. In centralized DCA schemes, a channel is selected for a new call from a central pool of free channels, and a particular characterizing function is utilized to select one of the available free channels. In distributed DCA schemes, a channel is selected for a new call from the cell associated with the call or from interfering neighboring cells. 
Centralized DCA schemes theoretically provide near optimal performance. However, using a centralized approach to determine the best available channel for a new call without causing any channel interference is a combinatorial optimization problem, which is computationally intractable. Therefore, most existing centralized DCA schemes are heuristic. For instance, Lima et al. presented a genetic algorithm (GA)-based DCA method [10]; Kim et al. proposed a DCA method that is based on the GA and minimizes inter-cell interference [11]; Misra et al. developed a learning automata-based channel reservation scheme to find the optimal number of reserved channels in a system [12]; Zhao et al. proposed a DCA method that is based on a noisy chaotic neural network [13]; Krishna et al. presented a dynamic channel allocation scheme with efficient channel reservation for handoff calls [14]. In all of the above approaches, the DCA is centrally implemented in the mobile switching center (MSC) or in a cell with a central controller, which collects information about all of the cells in the CMS. The shortcomings of such centralized schemes are as follows; (i) the MSC is increasingly likely to fail owing to an overload of call requests as the size of the CMS grows with the growth in the number of mobile users $[15,16]$, and (ii) determining the best available channel is time consuming even when heuristic methods are used, and a new call may be blocked as a result of just one unsuccessful assignment. These drawbacks suggest that distributed DCA schemes may be better for large CMS, because they assign and reassign channels at the base station of a cell $[17,18]$. Owing to the simplicity of the assignment algorithm at each base station, distributed DCA schemes are more attractive for implementation in a CMS. However, most current distributed DCA schemes impose only CCC. All current methods that deal with the CCC, ACC, and CSC simultaneously are centralized DCA schemes.

To maximize channel utilization, most of centralized DCA schemes account for the packing condition and the resonance condition in selecting a channel to be assigned for a new call. The packing condition is applied to minimize the number of channels that are used whenever a call arrives; it allows the selection of those channels that are already in use in other cells as long as the three types of constraint are satisfied. The resonance condition assigns the same channels to cells within the reuse distance without causing any channel interference. In centralized DCA schemes, these two conditions are incorporated into the objective function over all cells that are governed by the MSC or are put into typical compact patterns for reference in the assignment process. In the real word, the place and time of the arrival of a new call is unpredictable. Accordingly, the resonance condition is too ideal to occur, and the assignment based on the packing condition can hardly reach the resonance condition either. Thus, the effect of the channel utilization that arises from the assignment of centralized DCA schemes that are based on these two conditions is not as good as it seems. On the other hand, one drawback of distributed DCA schemes is lack of the overall information, which prevents distributed DCA schemes from performing the system-wise optimization, as is performed by centralized DCA schemes. However, this limitation requires researchers to develop innovative schemes for assigning an idle channel to an arriving new call and releasing an in-use channel when an ongoing call is completed in distributed DCA schemes. To overcome the aforementioned drawbacks of centralized DCA schemes, this work proposes a distributed dynamic channel assignment and reassignment (DDCAR) scheme. The proposed DDCAR scheme, which is the first contribution of this work, can satisfy the three types of constraint.

In a CMS, the service area is divided into cells, each of which is equipped with numerous channels, which are shared by two types of call - new calls and handoff calls. If a request for an arriving new call is denied, then this call will be blocked and cleared from the system. If a request for a handoff call is denied, then this call will be dropped immediately. Giving a higher priority to handoff calls than to new calls is common practice and is realized using a call admission control (CAC) strategy. CAC is such a provisioning strategy, which limits new calls into the system to reduce system congestion and the dropping of handoff calls. Good CAC strategies must balance new call blocking with handoff call dropping to satisfy quality of service (QoS) requirements. The earlier CAC algorithms [19-22] considered the effective traffic load of the target cell to determine whether the call should be accepted. However, these methods ignore the interference of to-be-accepted calls by the neighboring cells. A review of literature reveals that various CAC schemes depend on unrealistic, simplifying approximations and assumptions, resulting in imprecise control decisions [23]. Moreover, the computational complexity of heuristic-based CAC algorithms is very high because of the large number of states that describe the system. Fuzzy logic-based control schemes in CAC have been investigated [24] and found to be able to make intelligent control decisions successfully. Fuzzy logic can eliminate most of these assumptions and complicated computations because fuzzy if-then rules are based on linguistic variables that incorporate a human-like representation of information. Therefore, the second contribution of this work is a fuzzy call admission control (FCAC) scheme, combined with the DDCAR, for implementation at the base station. The proposed FCAC scheme not only controls the admission of new calls to reduce the dropping probability of handoff calls but also prevents channel interference. 
The paper is organized as follows. Section 2 describes the channel state table and presents the DDCAR schemes. Section 3 illustrates the FCAC scheme combined with the DDCAR. In Section 4, the DDCAR scheme is tested using extensive simulations to evaluate its performance and compared with two centralized DCA methods. Test results of the FCAC scheme are also presented in Section 4. Section 5 concludes the paper.

\section{Distributed dynamic channel assignment and reassignment}

\subsection{Cellular mobile system}

Hexagonal cells are widely used in CMS because they enable a geographic region to be covered with the fewest calls. Since each hexagonal cell has six equidistant neighbors and the lines that join the center of any cell to its neighbors are separated by multiples of $60^{\circ}$, only certain cluster sizes and cell layouts are permitted. Adjacent cells are connected without gaps when the geometry of the hexagons is such that the number of cells per cluster, $N$, satisfies the following equation.

$$
N=I^{2}+J^{2}+(I \times J) \quad I, J=0,1,2,3 .
$$

A large value of $N$ specifies that the ratio of the cell radius to the distance between co-channel cells is large and vice versa. The value of $N$ is a function of the degree of interference that can be tolerated by a mobile or base station while communications of sufficient quality are maintained.

The considered CMS is a 7-cell reuse cluster system as shown in Figure 1, such that the channel reuse distance is three cells. For example, in a portion of the CMS displayed in Figure 2, cells 2 to 7 are first-tier cells of cell 1, and cells 8 to 19 are the second-tier cells. The cells

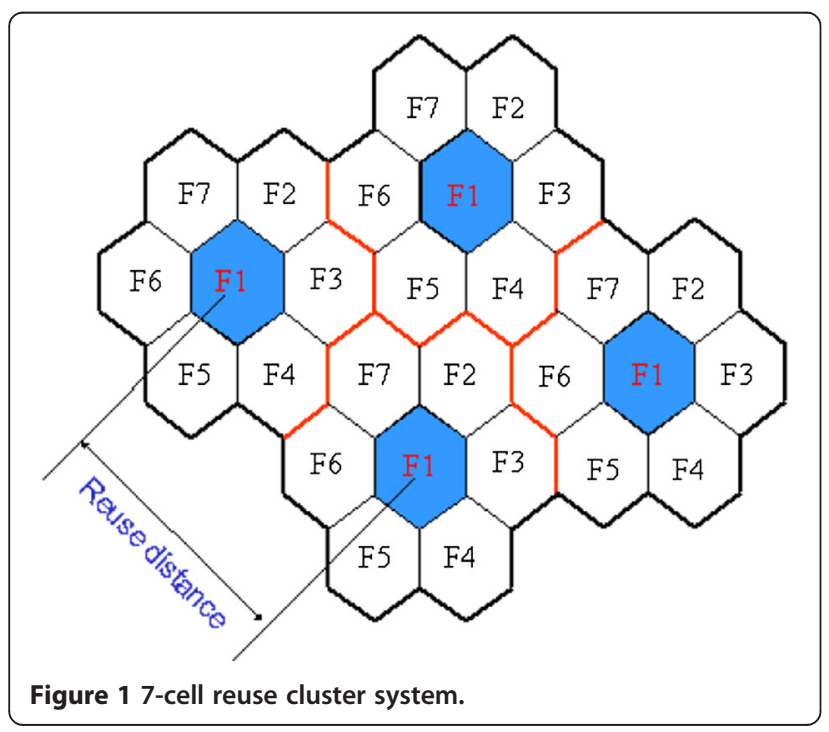

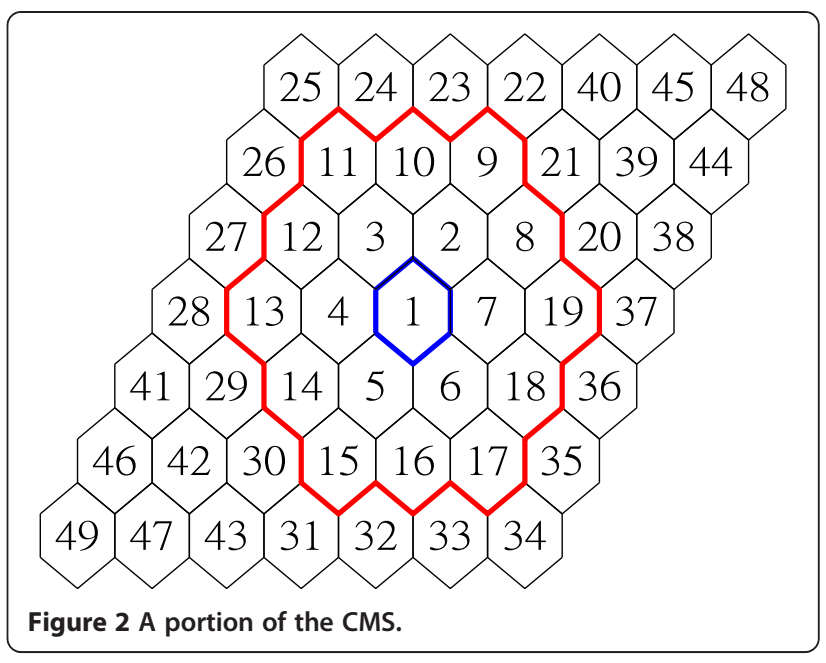

within the channel reuse distance for cell 1 are cells 2 to 19 as indicated within the bold boundary. Therefore, the third-tier cells 20 to 37 and the cells beyond can use the channels used in cell 1 . Let $C=\left\{c_{1}, c_{2}, \cdots, c_{k}, \cdots, c_{K}\right\}$ denote the set of channels, where the index $K$ denotes the identifier of the $k$ th channel. For simplicity, the adjacent channels of channel $c_{k}$ are assumed to be $c_{k-1}$ and $c_{k+1}$. Suppose channel $c_{k}$ is used in cell 1 , the CCC states that cells 2 to 19 cannot use channel $c_{k}$ that is currently used in cell 1 . Since the first-tier cells 2 to 7 are the adjacent cells of cell 1 , the ACC implies that they cannot use the adjacent channels $c_{k-1}$ and $c_{k+1}$ of the channel $c_{k}$. The CSC states that the channels used in the same cell should have a specified distance of two channels. Thus, if $c_{k}$ is used in cell $1, c_{k-2}$, $c_{k-1}, c_{k+1}$, and $c_{k+2}$ cannot be used in cell 1 .

As indicated in the preceding section, two types of call share the channels - new calls and handoff calls. New calls are those that are initiated by mobile users in the current cell, while handoff calls are those that were initiated in other cells and handed over to the current cell. When a call arrives at a cell in which no channel is available, it may be blocked or may be queued, depending on the CAC schemes used. The probability of a new call being blocked is called new call blocking probability $\left(P_{\mathrm{nb}}\right)$, and the probability of a handoff call being dropped is called handoff call dropping probability $\left(P_{\mathrm{hd}}\right)$. For a particular traffic load, $P_{\mathrm{nb}}$ and $P_{\mathrm{hd}}$ can be evaluated as follows:

$$
\begin{gathered}
P_{\mathrm{nb}}=\frac{B_{\mathrm{nb}}}{A_{\mathrm{nc}}} \\
P_{\mathrm{hd}}=\frac{D_{\mathrm{nh}}}{R_{\mathrm{ha}}}
\end{gathered}
$$

where $B_{\mathrm{nb}}$ is the number of new calls that are blocked, $D_{\text {nh }}$ is the number of handoff calls that are dropped, $A_{\text {nc }}$ 
is the number of new call arrivals, and $R_{\mathrm{ha}}$ is the number of handoff requests.

The values of $P_{\mathrm{nb}}$ and $P_{\mathrm{hd}}$ are determined by the design of the system. Each arriving call, regardless of whether it is a new call or a handoff call, will occupy a channel if it is served Accordingly, new calls and handoff calls compete for a finite number of channels in each cell. The trade-off between minimizing the probability of blocking a new call and minimizing the probability of dropping users in handover is well known. That is, $P_{\mathrm{nb}}$ and $P_{\mathrm{hd}}$ cannot be simultaneously reduced. From the perspective of users, the termination of a call during service is more troublesome than the blocking of a call, so $P_{\text {hd }}$ must be much lower than $P_{\mathrm{nb}}$. Therefore, handoff calls are frequently given a higher priority in accessing a channel.

With respect to call blocking, as the number of available channels in the system increases, the number of blocked calls decreases. Here, an available channel is an idle channel that can be assigned to a new call without causing any interference with the currently in-use channels in any cell. Accordingly, each cell requires only information about the channels in the cells within its channel reuse distance. A scheme that uses this information can be designed to select an available channel to be assigned to a new call with the purpose of minimizing the number of available channels that becomes unavailable within the system. Similarly, a scheme can also be designed to select an in-use channel to be released when a call completes under the condition of maximizing the number of unavailable channels that becomes available within the system. Meanwhile, the ongoing call that is associated with the released channel is reassigned to the channel that has just completed the call. This work firstly proposes a DDCAR scheme to select an available channel to be assigned to the new call and an in-use channel to be released upon call completion to satisfy the above three types of constraint simultaneously.

\subsection{Channel states and channel state table}

The DDCAR scheme in a cell is implemented as follows. If channels are available in the cell when a new call arrives, this new call will be accepted and assigned to the best available channel, according to the channel assignment criteria; otherwise, this new call is blocked. If other channels in a cell are in use when a call completes in that cell, then the channel reassignment criteria will identify the best in-use channel to be released and reassign the corresponding call to the channel in which the other call was just completed; otherwise, the channel in which a call was just completed will be released. The channel assignment criteria and channel reassignment criteria of the DDCAR scheme are developed to reduce the blocking probability, which will be presented in Section 2.3.

\subsubsection{Channel states}

We define $\{1,0,-1, \ldots,-n\}$ as the set of states for a channel. A value of ' 1 ' implies the channel is in - use. A value of ' 0 ' represents the channel is available for assignment. A value of ' -1 ' (or ' $-n$ ') indicates that if the considered channel is assigned to a new call, one (or $n$ ) currently in - use channel (s) in any cell will be interfered. For example, suppose channel $c_{k}$ is used in cell 1 , the state of channel $c_{k}$ in cell 1 is ' 1 ', while the states of channel $c_{k}$ in cells 2 to 19 are ' -1 ' as shown in Figure 3.

\subsubsection{Channel state table}

A cell will interfere with other cells only within its channel reuse distance. Therefore, each cell has a channel state table, which comprises the most updated states of all channels of the cells within the channel reuse distance. Table 1 presents an example of a channel state table with 12 cells and six channels: cell 1 represents the target cell, and the cells are numbered as in Figure 2. The channel states of all cells correspond to a time instance and satisfy definition of channel states and the three constraints, CCC, ACC, and CSC simultaneously. The states of channel 3 in cell 1 are ' 1 ', indicating that this channel is in use. Based on the CSC and definition of channel state, the states of both channels 1 and 2 in cell 1 should be smaller than or equal to '-1' depending on whether they are influenced by any other cells or not. In the current example, the state of channel 1 in cell 1 is ' -2 ', meaning that channels 1 and 3 affect this channel. Similarly, the states of channels 4 and 5 in cell 1 should be smaller than or equal to ' -1 '. Similar arguments apply to channel 4 in cell 1 ; the state of this channel is ' -2 ' rather than ' -1 ' owing to both CSC and ACC, because channel 5 is used in cell 7. The state of channel 6 in cell

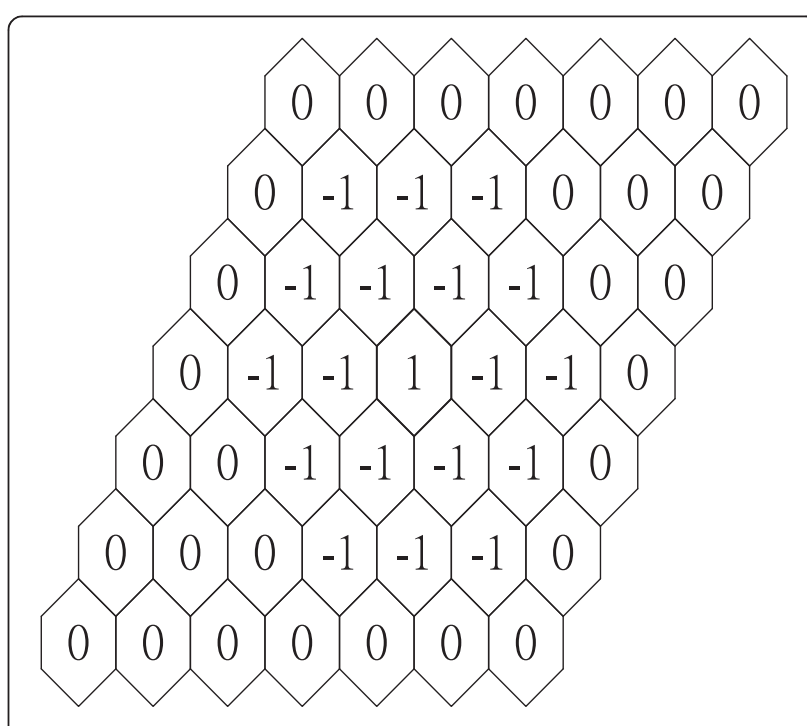

Figure 3 State of cells 1 to 19 when channel $c k$ is used in cell 1 . 
Table 1 A channel state table with 12 cells and six channels

\begin{tabular}{lllllll}
\hline Cell & \multicolumn{2}{l}{ Channel } & & & & \\
\cline { 2 - 6 } & $\mathbf{1}$ & $\mathbf{2}$ & $\mathbf{3}$ & $\mathbf{4}$ & $\mathbf{5}$ & $\mathbf{6}$ \\
\hline 1 & -2 & -1 & 1 & -2 & -1 & -2 \\
2 & 0 & -2 & 0 & -2 & -1 & -1 \\
3 & -1 & -1 & 0 & -1 & -2 & 0 \\
4 & 0 & -1 & 0 & -1 & -1 & -1 \\
5 & 0 & -1 & 0 & -1 & -1 & 0 \\
6 & -1 & -1 & 0 & -2 & 0 & -1 \\
7 & 0 & -3 & -1 & -2 & 1 & -2 \\
8 & 1 & -1 & -2 & -1 & 0 & -2 \\
9 & 0 & -1 & -1 & 0 & -2 & 0 \\
10 & -1 & 0 & -1 & -1 & -2 & 1 \\
11 & 0 & 0 & -1 & 0 & -1 & 0 \\
12 & 0 & 0 & -1 & 0 & 0 & -1 \\
\hline
\end{tabular}

1 is ' -2 ' owing to both CCC and ACC, because channel 10 is used in cell 6 and channel 5 is used in cell 7. Both cells 6 and 11 are within the channel reuse distance of cell 1, but as shown in Figure 2, they are separated from each other by three cells; so they can use the same channel at the same time without any interference. The state of channel 2 in cell 7 is ' -3 ', but this channel is observed to be affected by two currently in-use channels - channel 3 in cell 1 and channel 1 in cell 8 . This channel is also affected by an in-use channel in a particular cell (e.g., cell 20), which is not within the channel reuse distance of cell 1 , and is not shown in Table 1 .

\subsubsection{Evolution of channel states}

Whenever the channel state changes, the corresponding cell is assumed to inform immediately the other cells within its channel reuse distance. Then, the relevant cells can update their channel state tables accordingly. This assumption is reasonable because the transmission time between base stations is of the order of microsecond. Therefore, each cell will have an up-to-date channel state table at all times. The channel states of the system will change whenever an available channel is assigned to a new call or an in-use channel is released because a call is completed.

When the channel states of the system change because an available channel is assigned to a new call, the state of the assigned channel will change from ' 0 ' to ' 1 ', and the states of other affected channels will also change, as described below. Since only the channels of the cells within the channel reuse distance may undergo interference when a channel is assigned to a new call in the target cell, the region of interference that is generated owing to a channel assignment must firstly be identified in the channel state table. Table 2 presents an example of the channel state table with 19 cells and 12 channels, where cell 1 represents the target cell, and the numbering of all cells corresponds to that in Figure 2. Suppose channel 10 of cell 1 (the target cell) is selected to be assigned to a new call: the part that is enclosed by the bold boundary presented in Table 2 represents the region of interference as a result of this assignment, based on the CCC, ACC, and CSC. Now, if channel 10 of cell 1 is assigned to a new call, then its state will change from ' 0 ' to ' 1 ', and the values of the states of the channels within the interference region will be decreased by 1 , such that if the state of a channel is ' 0 ' (or ' $-n$ '), then it will become ' -1 ' ( or ' $-\mathrm{n}-1$ ').

When a call completes in a cell, a selected in-use channel will be released. The state of the released in-use channel will change from ' 1 ' to ' 0 ', and the values of the states of the channels in the region of interference of the released channel will be increased by 1 , such that if the state of a channel is ' -1 ', then it will become ' 0 '.

\subsection{DDCAR scheme}

\subsubsection{Channel assignment criteria}

The basic idea of the channel assignment criteria is to identify a channel using the channel state table, such that the assignment thereof to the new call will cause

Table 2 A channel state table with 19 cells and 12 channels

\begin{tabular}{lllllllllllll}
\hline Cell & \multicolumn{1}{l}{ Channel } & & & & & & & & & & \\
\cline { 2 - 10 } & $\mathbf{1}$ & $\mathbf{2}$ & $\mathbf{3}$ & $\mathbf{4}$ & $\mathbf{5}$ & $\mathbf{6}$ & $\mathbf{7}$ & $\mathbf{8}$ & $\mathbf{9}$ & $\mathbf{1 0}$ & $\mathbf{1 1}$ & $\mathbf{1 2}$ \\
\hline 1 & -1 & -3 & 1 & -2 & -2 & 1 & -2 & -2 & -1 & 0 & 0 & -2 \\
2 & -1 & -2 & -2 & -1 & -1 & -1 & -1 & -2 & 0 & 0 & -1 & -1 \\
3 & 0 & -2 & -1 & -1 & -1 & -1 & -1 & -1 & 0 & 0 & -1 & -1 \\
4 & 0 & -2 & -1 & -1 & -1 & -1 & -2 & -1 & -1 & 0 & 0 & -2 \\
5 & -1 & -2 & -2 & -1 & -1 & -2 & -2 & 1 & -1 & -1 & -1 & -1 \\
6 & -1 & -3 & -2 & -1 & -1 & -1 & -2 & -1 & -1 & 0 & -1 & -1 \\
7 & -1 & -3 & -2 & -1 & -1 & -1 & -1 & -1 & 0 & 0 & 0 & -2 \\
8 & -1 & 1 & -2 & -1 & 0 & -1 & 0 & 0 & 0 & 0 & 0 & -1 \\
9 & -1 & -1 & -2 & 0 & 0 & -1 & 0 & 0 & 0 & 0 & -1 & -1 \\
10 & 0 & -1 & -1 & 0 & 0 & -1 & 0 & -1 & 0 & -1 & -1 & 1 \\
11 & 0 & 0 & -1 & 0 & 0 & -1 & 0 & 0 & 0 & 0 & -1 & -1 \\
12 & 0 & 0 & -1 & 0 & 0 & -1 & 0 & -1 & 0 & 0 & 0 & -1 \\
13 & 0 & 0 & -1 & 0 & 0 & -1 & 0 & -1 & 0 & 0 & 0 & 0 \\
14 & 0 & -1 & -1 & 0 & 0 & -1 & -1 & -1 & -1 & 0 & 0 & -1 \\
15 & -1 & -1 & -2 & -1 & 0 & -1 & -1 & -1 & -1 & 0 & -1 & -1 \\
16 & -1 & 1 & -2 & -1 & 0 & -1 & -1 & -1 & -1 & -1 & -1 & 1 \\
17 & -1 & -1 & -3 & 0 & 0 & -1 & 0 & -1 & 0 & 0 & -1 & -1 \\
18 & 0 & -2 & -1 & 0 & 0 & -1 & 0 & -1 & 0 & 0 & 0 & -1 \\
19 & -1 & -1 & -2 & 0 & 0 & -1 & 0 & 0 & 0 & 0 & 0 & 0 \\
\hline
\end{tabular}


the smallest possible number of available channels in the system to become unavailable. Therefore, when a new call arrives, the available channel with the fewest '0's within its interference region will be assigned. As explicated in Section 2.2.3, if an available channel is assigned to a new call, then the state of that channel changes from ' 0 ' to ' 1 ', and the values of the states of the channels in the interference region will be decreased by 1 . The change of the state of a channel from ' 0 ' to ' -1 ' means that an available channel has become unavailable. For example, the number of available channels in the system that becomes unavailable owing to the assignment of channel 10 in cell 1 to a new call is the number of '0's within its interference region, as shown in Table 2. Therefore, the criteria for assigning a channel to a newly arriving call are stated as follows. When a new call arrives, the target cell will set up a priority list for all of the available channels, which are those in the second row of Table 2 with state ' 0 '. The priority of an available channel is determined from the number of 'O's within its interference region: a channel with fewer zeros has higher priority. Therefore, the available channel that has the highest priority is the one whose assignment to a new call causes the fewest available channels to become unavailable.

Based on the assumption that all cells within the channel reuse distance will be informed immediately of a change of a state in any cell in that channel, the list of priorities of all the available channels can be updated whenever the state table changes. The channel with the highest priority in the priority list of all available channels, which is based on the channel assignment criteria, will be assigned. In Table 2, the interference regions of available channels 10 and 11 in cell 1 have 21 and 14 '0's in, respectively. Therefore, channel 11 in cell 1 has higher priority and is selected for assignment. However, if multiple channels in the same cell have the same priority, meaning that all have the fewest ' 0 's, the one with smaller channel index is chosen.

\subsubsection{Channel reassignment criteria}

When an ongoing call completes, a channel is released. However, the channel that was used by the justcompleted call does not have to be released. If other calls are ongoing in the same cell, then an in-use channel whose release will make more unavailable channels become available can be released and the channel that was used by the just-completed call can be assigned to that ongoing call. As described in Section 2.2.3, the state of the released channel then changes from ' 1 ' to ' 0 ', and the values of the states of the channels in the interference region of the released channel will be increased by 1. The change of state of a channel from ' -1 ' to ' 0 ' means that the channel, previously unavailable, has become available. Therefore, to identify the best in-use channel to release, the criteria for reassigning a channel upon the completion of an ongoing call are as stated below. The priority of an in-use channel is determined from the number of '-1's within its interference region: the priority increases with the number of ' -1 's. Therefore, the in-use channel with the highest priority is the one whose release will cause the most unavailable channels to become available.

Similar to the priority list of all the available channels, the in-use channels can be maintained and updated whenever the channel state table is changed. The channel with the highest priority in the priority list of all the in-use channels, prepared by applying the channel reassignment criteria, is selected to be released, and the call that is associated with that channel will be reassigned to the channel of the justcompleted call. For example, suppose the call that uses channel 3 in cell 1 completes, the number of ' -1 's within the interference regions of channel 3 is 15 , as indicated in the part that is enclosed by the double line boundary presented in Table 2. Since channel 6 in cell 1 is also in use, the number of ' -1 's within the interference regions of channel 6 is 26 . Thus, channel 3 is assigned to the ongoing call that is using channel 6 and channel 6 is released, releasing 26 cells. However, multiple in-use channels have the same number of ' -1 's in their interference regions; then, the channel with the larger channel index will be assigned to the call. The channel reassignment criteria are indeed the opposite of the channel assignment criteria.

\section{Fuzzy call admission control}

The proposed FCAC scheme is based on effective traffic loads for both the target cell and the adjacent cells. Because when the system is heavily loaded, the acceptance of a new call in one cell may cause dropping or outage in adjacent cells. Thus, the goal of the FCAC scheme is to significantly reduce the dropping probability at the cost of increasing the blocking probability to an acceptable level.

\subsection{FCAC scheme combined with the DDCAR}

The FCAC scheme, combined with the DDCAR, is implemented in three steps.

Step 1 If the number of available channels in the target cell is zero, then neither an arriving new call nor a handoff call may be served. The new call is blocked, and the handoff call is dropped immediately.

Step 2 If the number of available channels in the target cell exceeds zero and the request is a handoff call, then that call is served. The best available channel, based on the channel reassignment criteria, is reassigned to this call.

Step 3 If the number of available channels in the target cell exceeds zero and the request is a new call, 
fuzzy inference system (FIS) [25,26] determines whether this call is accepted. If the FIS allows this new call to be served, then the best available channel, based on the channel assignment criteria, is assigned to this call; otherwise, the call is blocked.

\subsection{Fuzzy inference system}

An FIS is a rule-based architecture that comprises four principal parts, which are a fuzzifier, a fuzzy rule base, an inference engine, and a defuzzifier. The fuzzifier converts a crisp set of input data into input linguistic values using fuzzy linguistic variables, fuzzy linguistic terms, and membership functions. The fuzzy rule base stores empirical knowledge of domain experts concerning. After fuzzification, the inference engine refers to the fuzzy rule base, which contains fuzzy IF-THEN rules, to derive linguistic values of the intermediate and output linguistic variables. When the output linguistic values have become available, the defuzzifier generates the final crisp values from the output linguistic values.

Typically, a membership function is used to fuzzify input and output variables. Most such membership functions of parameters are triangular; these are used herein. The FIS yields the number of available channels, the blocking probability, and the dropping probability to control the admission of a new call in a manner determined by the traffic load. Therefore, the FIS has three input variables, $x_{1}, x_{2}$, and $x_{3}$, and one output variable, $y$, which are defined as follows. Let $X_{1}$ denote the number of available channels and $x_{1}$ represent the normalized value of the available channels. Suppose the total number of channels in the system is $T C$, the normalized value of the available channels is $x_{1}=X_{1} /$ $T C$. Let $x_{2}$ and $x_{3}$ represent the average dropping probability and the average blocking probability, respectively, over the preceding $20 \mathrm{~min}$. The output variable $y$ denotes the probability that a new call will be permitted. A large $y$ specifies a high rate of acceptance of new calls by the FIS. The fuzzy linguistic expressions of input and output system variants are given in Table 3.

Figure 4 shows the block diagram of the FIS. The membership functions of input and output parameters are abbreviated and shown in the Figures 5, 6, 7 and 8. In membership function of $x_{1}, \mathrm{~S}$ stands for small, $\mathrm{M}$ for middle, and $\mathrm{B}$ for big. In membership functions of $x_{2}$

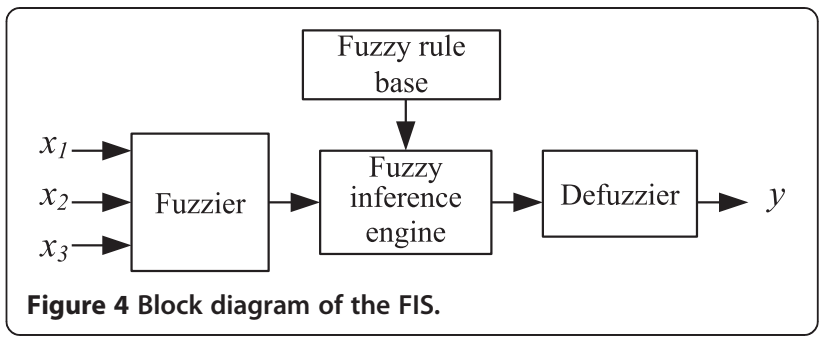

and $x_{3}$, L stands for low, $\mathrm{M}$ for medium, and $\mathrm{H}$ for high. In membership function of $y, \mathrm{~L}$ stands for low, ML for medium low, $\mathrm{MH}$ for medium high, and $\mathrm{H}$ for high.

\subsection{Remark 1}

The values of lower limit $=0$, center $=0.2$, and upper limit $=0.4$ for both $x_{2}$ and $x_{3}$ are obtained from the results of empirical tests using the test example that was described in Section 4.1. Most network regulators require a dropping probability of equal to or less than $3 \%$ and blocking probability of equal to or less than $5 \%$, to ensure QoS performance. However, a $7 \times 7$ parallelogram-shaped CMS with a 7 -cell reuse cluster is considered herein and three EMC constraints are simultaneously imposed under a high traffic load. Clearly, a larger channel reuse distance causes fewer channels to be reused throughout the service area. Section 4.2 investigated the effect of the three EMC constraints on the blocking probability and the dropping probability and presented that these three EMC constraints greatly worsen the performance of channel assignment methods. When the increase in traffic load exceeded $40 \%$, the blocking probability exceeded its recommended operating value of $5 \%$. Finally, high traffic loads were used to test the robustness of the proposed method. Under high traffic load, few resources are available and call requests tend to be blocked or dropped. The blocking probability and dropping probability tend to increase significantly when the traffic overload is very large owing to the exhaustion of system resources.

To construct the rule base of the fuzzy model, 27 rules were developed, based on data sets and the experiences of experts. The fuzzy IF-THEN rules composed by the FIS are given in Table 4 . These 27 rules are applied to the FIS. Since the Mamdani fuzzy inference mechanism is commonly used to develop fuzzy models [25], it is

Table 3 The fuzzy linguistic expressions of input/output system variants

\begin{tabular}{llll}
\hline Notation & Parameters & Type & Linguistic expressions \\
\hline$x_{1}$ & Normalized value of the available channels & Input & B (big), M (middle), S (small) \\
$x_{2}$ & Average dropping probability & Input & H (high), M (medium), L (low) \\
$x_{3}$ & Average blocking probability & Input & $H$ (high), M (medium), L (low) \\
$y$ & Rate of acceptance of new calls & Output & H (high), MH (medium high), ML (medium low), L (low) \\
\hline
\end{tabular}




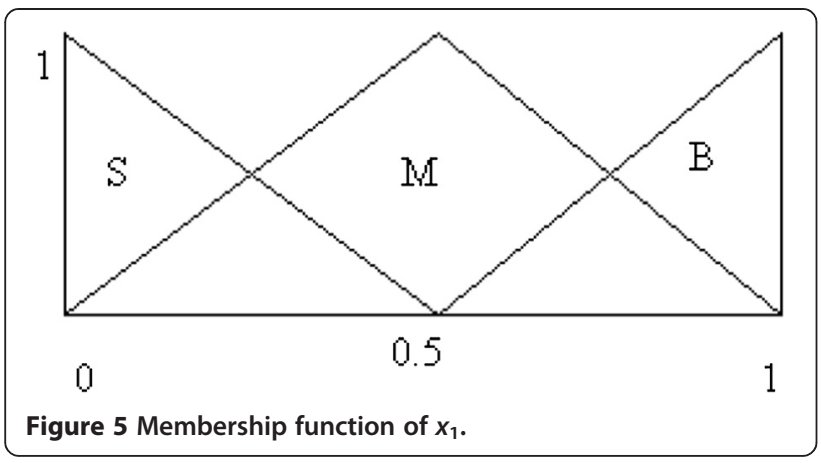

used herein and specifies output variables as fuzzy sets. This approach uses the max-min composition [27], which is described as following.

$$
\mu_{D_{k}}(y)=\max _{k}\left[\min \left[\mu_{A_{k}}\left(x_{1}\right), \mu_{B_{k}}\left(x_{2}\right), \mu_{C_{k}}\left(x_{3}\right)\right]\right], k=1, \ldots, 27 .
$$

where $\mu_{D_{k}}, \mu_{A_{k}}, \mu_{B_{k}}$, and $\mu_{C_{k}}$ are the membership functions of output $y$ and inputs $x_{1}, x_{2}$, and $x_{3}$ of the $k$ th rule, respectively.

The rules are disjunctive, so the aggregation operation 'max' results in an aggregated membership function that comprises the outer envelope of the individual truncated membership forms for each rule. If the aggregated output required a crisp value, suitable defuzzification techniques must be utilized in the aggregated membership function. Defuzzification refers to the method by which a crisp value is extracted from a fuzzy set as a representative value. The five general defuzzification methods are centroid of area (COA), center of gravity, mean of maximum, smallest of maximum, and largest of maximum. The most commonly used method is COA [27], which is described as follows.

$$
y^{*}=\frac{\int_{Y} \mu_{D}(y) \cdot y d y}{\int_{Y} \mu_{D}(y) d y}
$$

When the value of $y^{*}$ has been obtained, the acceptance of a new call is according to the following criteria. If $y^{*} \geq \alpha$,

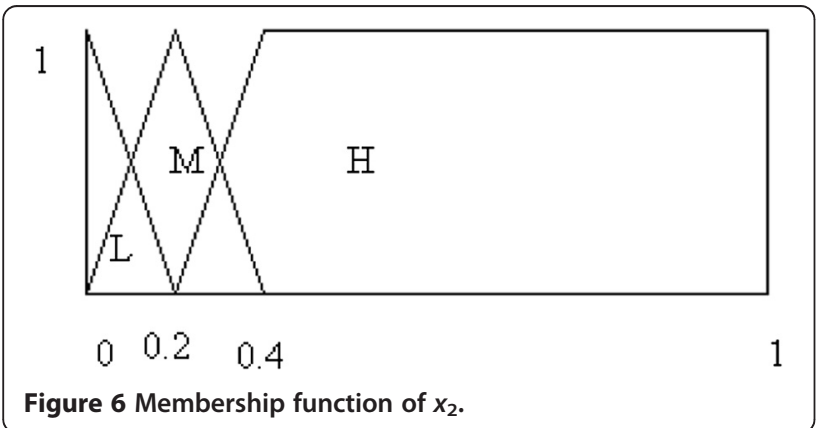

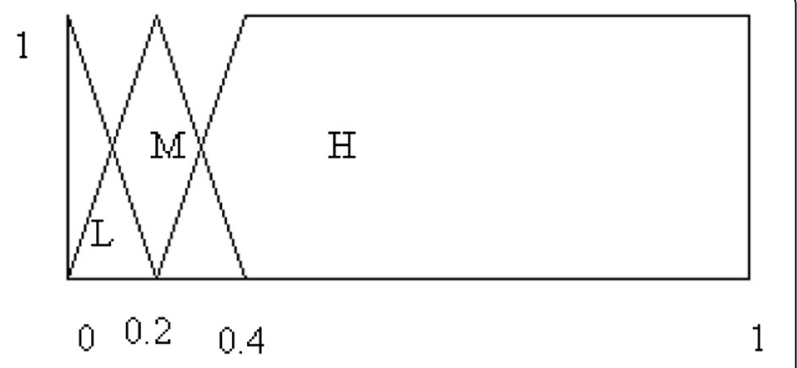

Figure 7 Membership function of $x_{3}$.

where $\alpha$ is a predefined threshold value in the range from 0 to 1 , then the new call is accepted for service and the best available channel, determined using the channel assignment criteria, is assigned to it. If $y^{*}<\alpha$, then the new call is not served and it is blocked immediately.

\section{Test results and comparison}

\subsection{Simulation model and assumptions}

A $7 \times 7$ parallelogram-shaped CMS with 7 -cell reuse cluster is employed as the test example, as shown in Figure 2. The total number of channels in the system is assumed to be 70, and each channel can serve only one call. The three types of constraint, CCC, ACC, and CSC, are all taken into account. The call arrivals are assumed to follow a Poisson process with the distribution for inter-arrival time as $e^{-\lambda t}$, where $\lambda$ represents the mean of call arrival rate. The call holding time is assumed to follow an exponential distribution $e^{-\lambda t}$, where $\mu$ represents the mean of call holding time that equals to $180 \mathrm{~s}$ throughout the simulations.

Figure 9 shows the cellular environment using two nonuniform traffic patterns which are constant-rate voice calls, denoted by traffic patterns A and B, respectively [10]. Traffic patterns A and B represent the light (average of 91.83 calls per hour) and heavy (average of 106.53 calls per hour) traffic load, respectively. The numbers with unit calls/hour shown in each cell denote the initial mean of call arrival rate. The performance measure used herein is the blocking probability of incoming calls across the entire CMS. To evaluate the performance of the DDCAR scheme and to

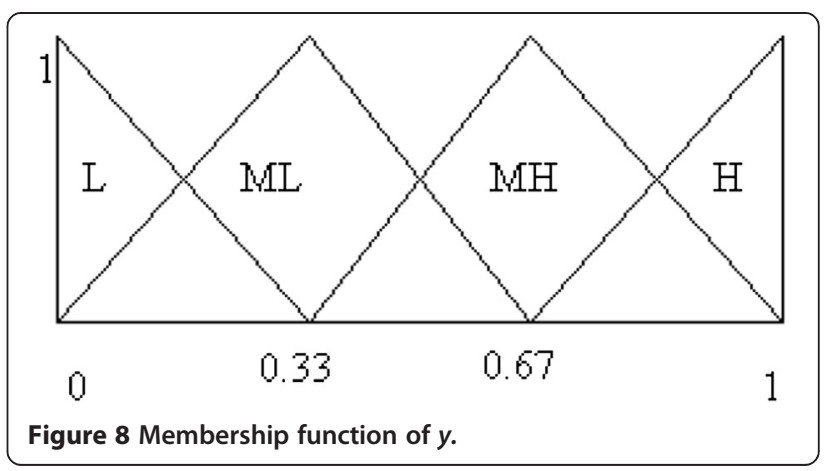


Table 4 The fuzzy IF-THEN rules composed by the FIS

\begin{tabular}{|c|c|}
\hline Rule number & Description of IF-THEN rules \\
\hline 1 & If $x_{1}$ is $B, x_{2}$ is $H$, and $x_{3}$ is $H$, then $y$ is $L$. \\
\hline 2 & If $x_{1}$ is $B, x_{2}$ is $H$, and $x_{3}$ is $M$, then $y$ is $L$. \\
\hline 3 & If $x_{1}$ is $B, x_{2}$ is $H$, and $x_{3}$ is $L$, then $y$ is $M L$. \\
\hline 4 & If $x_{1}$ is $B, x_{2}$ is $M$, and $x_{3}$ is $H$, then $y$ is $M L$. \\
\hline 5 & If $x_{1}$ is $B, x_{2}$ is $M$, and $x_{3}$ is $M$, then $y$ is $M H$. \\
\hline 6 & If $x_{1}$ is $B, x_{2}$ is $M$, and $x_{3}$ is $L$, then $y$ is $M H$. \\
\hline 7 & If $x_{1}$ is $B, x_{2}$ is $L$, and $x_{3}$ is $H$, then $y$ is $L$. \\
\hline 8 & If $x_{1}$ is $B, x_{2}$ is $L$, and $x_{3}$ is $M$, then $y$ is $M H$. \\
\hline 9 & If $x_{1}$ is $B, x_{2}$ is $L$, and $x_{3}$ is $L$, then $y$ is $H$. \\
\hline 10 & If $x_{1}$ is $M, x_{2}$ is $H$, and $x_{3}$ is $H$, then $y$ is $L$. \\
\hline 11 & If $x_{1}$ is $M, x_{2}$ is $H$, and $x_{3}$ is $M$, then $y$ is $L$. \\
\hline 12 & If $x_{1}$ is $M, x_{2}$ is $H$, and $x_{3}$ is $L$, then $y$ is $L$. \\
\hline 13 & If $x_{1}$ is $M, x_{2}$ is $M$, and $x_{3}$ is $H$, then $y$ is $L$. \\
\hline 14 & If $x_{1}$ is $M, x_{2}$ is $M$, and $x_{3}$ is $M$, then $y$ is $L$. \\
\hline 15 & If $x_{1}$ is $M, x_{2}$ is $M$, and $x_{3}$ is $L$, then $y$ is $M L$. \\
\hline 16 & If $x_{1}$ is $M, x_{2}$ is $L$, and $x_{3}$ is $H$, then $y$ is $L$. \\
\hline 17 & If $x_{1}$ is $M, x_{2}$ is $L$, and $x_{3}$ is $M$, then $y$ is $M L$. \\
\hline 18 & If $x_{1}$ is $M, x_{2}$ is $L$, and $x_{3}$ is $L$, then $y$ is $H$. \\
\hline 19 & If $x_{1}$ is $S, x_{2}$ is $H$, and $x_{3}$ is $H$, then $y$ is $L$. \\
\hline 20 & If $x_{1}$ is $S, x_{2}$ is $H$, and $x_{3}$ is $M$, then $y$ is $L$. \\
\hline 21 & If $x_{1}$ is $S, x_{2}$ is $H$, and $x_{3}$ is $L$, then $y$ is $L$. \\
\hline 22 & If $x_{1}$ is $S, x_{2}$ is $M$, and $x_{3}$ is $H$, then $y$ is $L$. \\
\hline 23 & If $x_{1}$ is $S, x_{2}$ is $M$, and $x_{3}$ is $M$, then $y$ is $L$. \\
\hline 24 & If $x_{1}$ is $S, x_{2}$ is $M$, and $x_{3}$ is $L$, then $y$ is $M L$. \\
\hline 25 & If $x_{1}$ is $S, x_{2}$ is $L$, and $x_{3}$ is $H$, then $y$ is $L$. \\
\hline 26 & If $x_{1}$ is $S, x_{2}$ is $L$, and $x_{3}$ is $M$, then $y$ is $M L$. \\
\hline 27 & If $x_{1}$ is $S, x_{2}$ is $L$, and $x_{3}$ is $L$, then $y$ is $M H$. \\
\hline
\end{tabular}

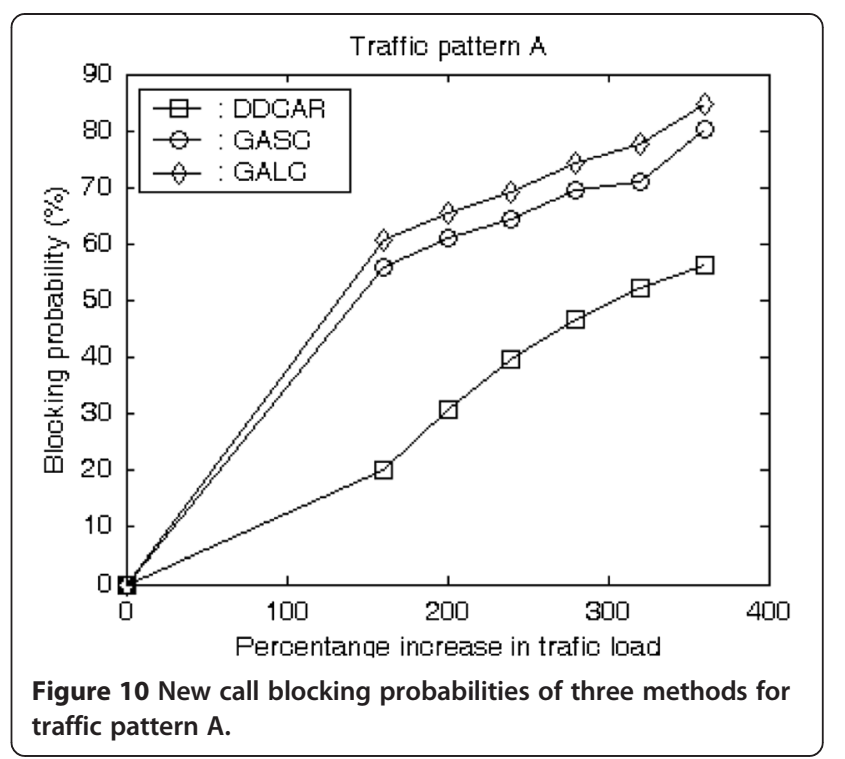

compare methods under various traffic loads, the traffic load was increased by a percentage factor ranging $160 \%$ to $360 \%$. A percentage increase in traffic load is by definition the increase in the initial call arrival rates across each cell.

\subsection{Simulation results of the DDCAR}

For each traffic pattern and each traffic load, the proposed DDCAR scheme was simulated over $20 \mathrm{~h}$. The resulting blocking probabilities are presented in Figures 10 and 11 (marked by 'square' symbols) for traffic patterns A and B, respectively. In the meantime, the corresponding average number of available channels left in the system counted at every 100 calls is also recorded in Figures 12 and 13 (also marked by 'square' symbols) for traffic patterns A and B, respectively.

Two GA-based methods, GALC and GASC [10], are compared. The difference between GALC and GASC is that when a new call arrives, the former only selects a channel for the new call, whereas the latter reassigns channels to all ongoing calls, including the new call. Therefore, GASC outperforms GALC but requires more
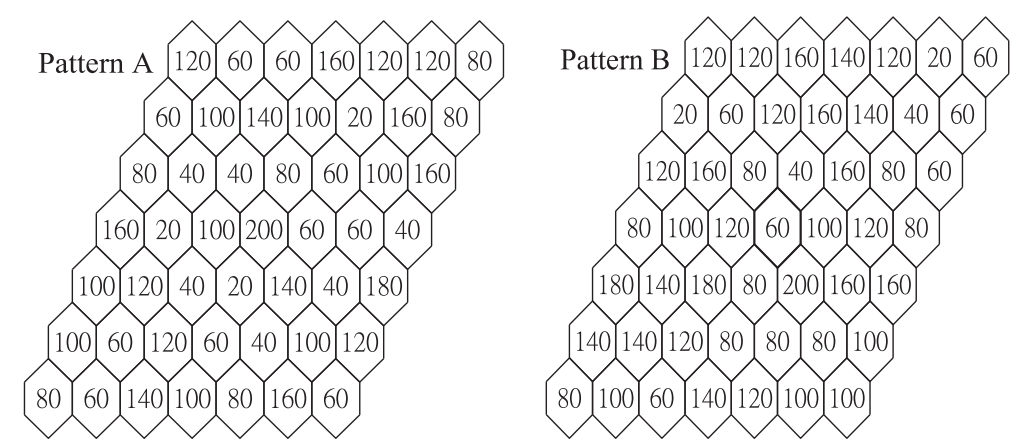

Figure 9 A $7 \times 7$ cellular environment with traffic patterns A and B. 


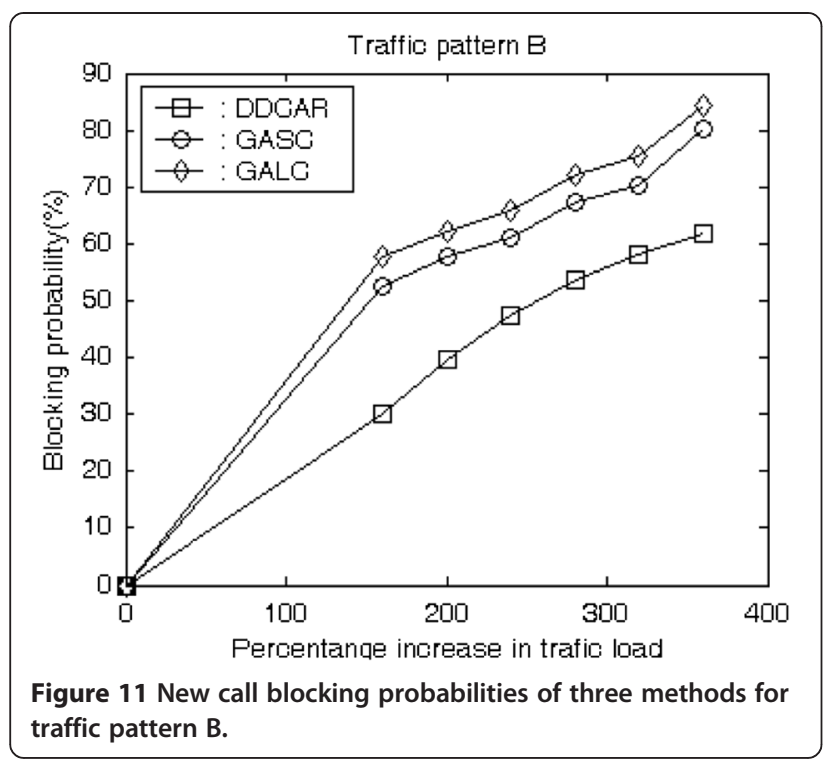

computing time. For each traffic pattern and each traffic load, GALC and GASC were simulated over $20 \mathrm{~h}$. The blocking probabilities and the average number of available channels in the system, determined at every 100 calls, obtained by these methods are marked by 'diamond' and 'circle' symbols, respectively, in Figures 10, 11, 12, and 13. According to Figures 12 and 13, we see that the DDCAR scheme yields more available channels than GALC and GASC, revealing that the DDCAR scheme yields better blocking probabilities than the other methods, as shown in Figures 10 and 11. As expected, GASC outperforms GALC, as demonstrated in Figures 10, 11, 12, and 13.

Notably, the above simulations are carried out in a Pentium-IV 20-GHz and 1.00 GB RAM personal computer. The central-processing-unit time consumed by the DDCAR scheme for each channel assignment and channel

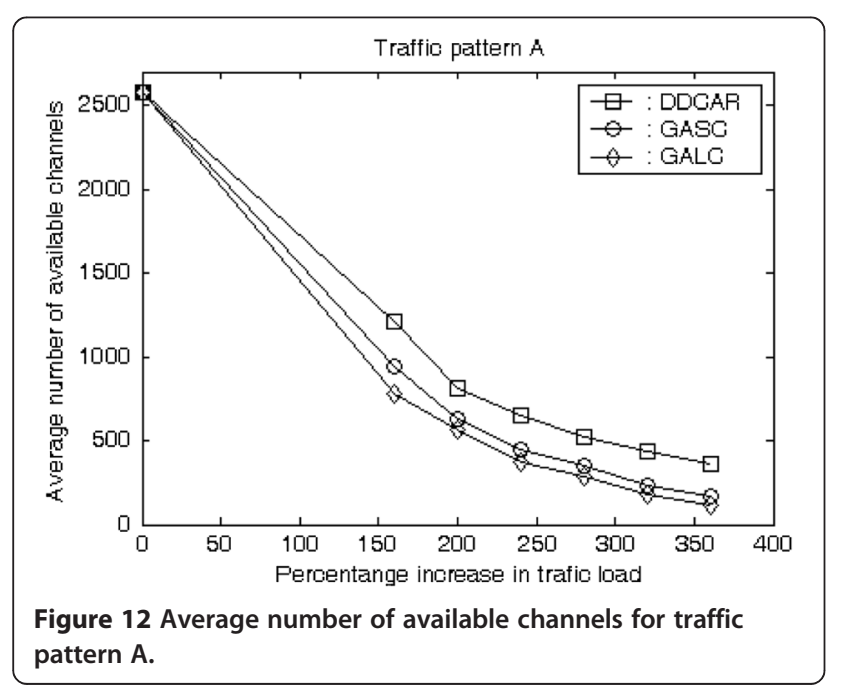

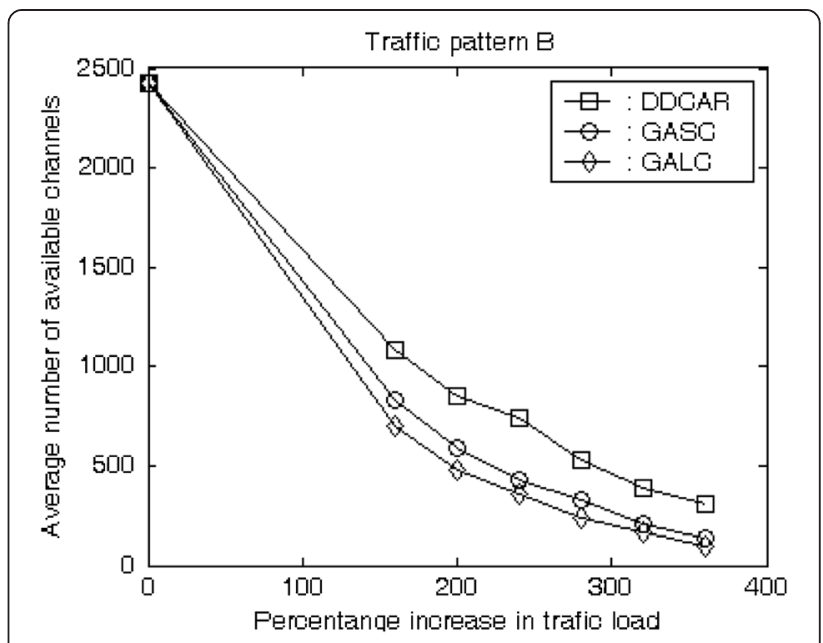

Figure 13 Average number of available channels for traffic pattern B.

reassignment is $2.3156 \times 10^{-4} \mathrm{~s}$ on the average, which is extremely faster than any centralized DCA method.

\subsection{Simulation results of FCAC}

To assess the performance of the FCAC and only the DDCAR under various traffic loads, the traffic load is increased by a percentage factor from $120 \%$ to $400 \%$ with two traffic patterns. The performance measure is the dropping probability of handoff calls. For each traffic pattern and each traffic load, the two methods were simulated over $20 \mathrm{~h}$. Two threshold values $\alpha$ are used in the FCAC scheme. Case I uses $\alpha=0.5$, and case II uses $\alpha=$ rand $[0,1]$, where rand $[0,1]$ is a uniformly distributed random number in the range $[0,1]$. For traffic pattern $\mathrm{A}$, the blocking probabilities and the dropping probabilities that were obtained by the FCAC scheme in cases I and II and those obtained using only the DDCAR are

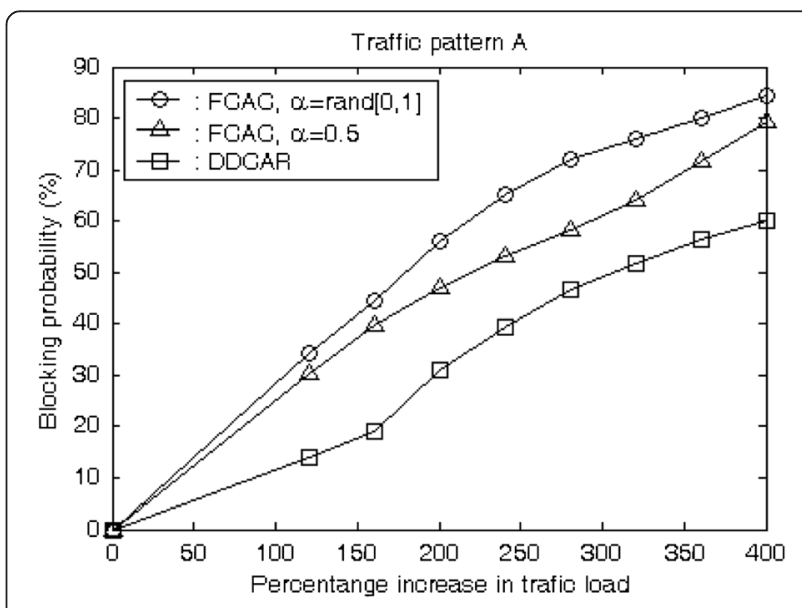

Figure 14 Blocking probabilities of DDCAR with and without FCAC for traffic pattern A. 


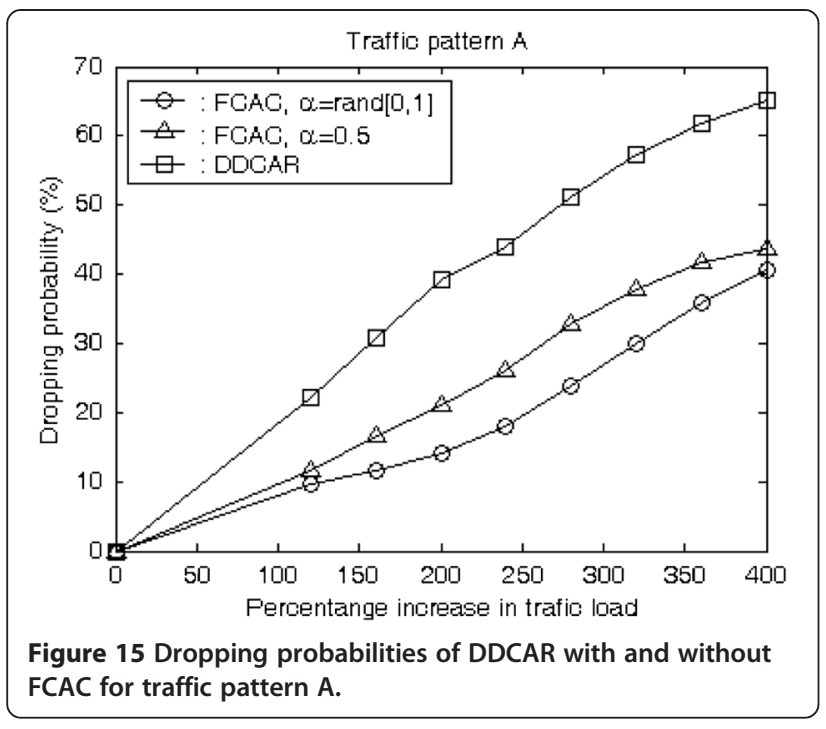

marked by 'triangle', 'circle', and 'square' symbols, respectively, in Figures 14 and 15. The results of traffic pattern B are displayed in Figures 16 and 17.

According to Figures 15 and 17, the dropping probabilities that were obtained in both cases by the FCAC scheme are lower than those obtained using only the DDCAR. The blocking probabilities in both cases that were obtained using the FCAC scheme exceed those obtained using only the DDCAR. Furthermore, the FCAC scheme in case II can further decrease the dropping probability. The cost is the increase of the blocking probability. By integrating the FCAC with DDCAR, the admission policy in case II for new calls becomes stricter. A new call can be admitted only when the admission criteria in the FIS is met. More new calls are blocked to reserve more channels to accommodate more future handoff calls. Handoff calls are given even higher priority, which

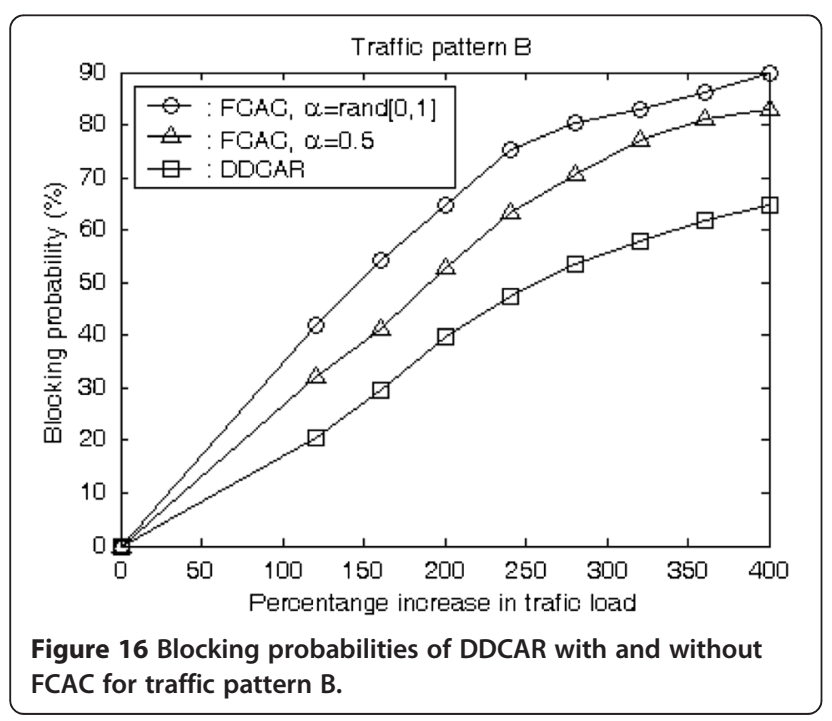

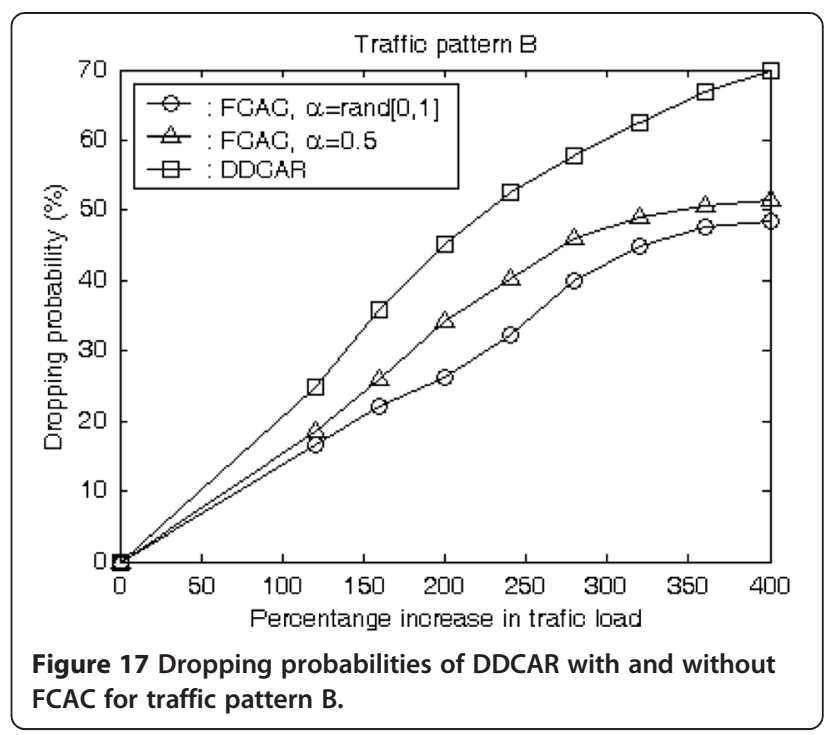

means that once a call is admitted, it can obtain a better service.

\section{Conclusions}

In a CMS, three types of interference, CCC, ACC, and CSC, are generally considered and two types of call - new calls and handoff calls - share the channels. Distributed DCA is the currently used channel assignment scheme for CMS because the demand of mobile users is growing. This work proposes a DDCAR scheme to maximize channel utilization. The DDCAR scheme has two novel features: (i) it satisfies CCC, ACC, and CSC simultaneously, and (ii) it exhibits the dynamic channel assignment criteria and channel reassignment criteria. Although each cell has access only to information about the channels in cells within the channel reuse distance, the DDCAR scheme yields a better blocking probability than the centralized DCA methods. Giving a higher priority to handoff calls than new calls is common practice, which can be realized using the CAC strategy. To give handoff calls a higher priority, a scheme that combines FCAC with DDCAR is proposed for execution at the base station. The FCAC scheme not only controls the admission of new calls to reduce the dropping probability but also prevents channel interference. Test results reveal that the FCAC scheme provides a significantly lower dropping probability; however, but at the price of an increased blocking probability.

\section{Competing interests}

The authors declare that they have no competing interests.

\section{Authors' information}

S-CH is currently an associate professor of the Department of Computer Science and Information Engineering at Chaoyang University of Technology, Taiwan, Republic of China. S-SL is now a professor of the Department of Electrical Engineering at St. John's University, Taiwan, Republic of China. 


\section{Acknowledgements}

This research work is supported in part by the Ministry of Science and Technology in Taiwan, Republic of China, under Grant MOST103-2221-E-324-038, MOST103-2221-E-129-005, and NSC103-2623-E-129-002-ET.

\section{Author details}

${ }^{1}$ Department of Computer Science and Information Engineering, Chaoyang University of Technology, 168 Jifong E. Rd., Wufeng District, Taichung City 41349, Taiwan. ${ }^{2}$ Department of Electrical Engineering, St. John's University, 499, Sec. 4 Tam King Road, Tamsui, Taipei 25135, Taiwan.

Received: 29 September 2014 Accepted: 10 March 2015

Published online: 24 March 2015

\section{References}

1. ZM Loni, NM Khan, Analysis of fading statistics in cellular mobile communication systems. J Supercomput 64(2), 295-309 (2013)

2. M Canales, JR Gallego, A Hernandez, A Valdovinos, An adaptive location management scheme for mobile broadband cellular systems. Telecommun Syst 52(1), 299-315 (2013)

3. DL Hu, SW Mao, On co-channel and adjacent channel interference mitigation in cognitive radio networks. Ad Hoc Netw 11(5), 1629-1640 (2013)

4. GS Uyanik, MJ Abdel-Rahman, M Krunz, Optimal channel assignment with aggregation in multi-channel systems: a resilient approach to adjacent-channel interference. Ad Hoc Netw 20, 64-76 (2014)

5. GK Audhya, K Sinha, SC Ghosh, BP Sinha, A survey on the channel assignment problem in wireless networks. Wirel Commun Mob Comput 11(5), 583-609 (2011)

6. MP Mishra, PC Saxena, Survey of channel allocation algorithms research for cellular systems. Int J Netw Commun 2(5), 75-104 (2012)

7. GK Audhya, K Sinha, K Mandal, R Dattagupta, SC Ghosh, BP Sinha, A new approach to fast near-optimal channel assignment in cellular mobile networks. IEEE Trans Mob Comput 12(9), 1814-1827 (2013)

8. H Al-Mahdi, H Nassar, Y Fouad, M Ali, Design and analysis of a channel assignment scheme for CDMA/TDMA mobile networks. Wirel Pers Commun 69(2), 579-599 (2013)

9. D Agrawal, QA Zeng, Introduction to Wireless and Mobile Systems, 3rd edn. (Cengage Learning, Stamford, CT, 2011)

10. MAC Lima, AFR Araujo, AC Cesar, Adaptive genetic algorithms for dynamic channel assignment in cellular mobile communication systems. IEEE Trans Veh Technol 56(5), 2685-2696 (2007)

11. HS Kim, DH Kim, Genetic algorithm-based dynamic channel allocation to minimize the inter-cell interference in downlink wireless communication systems. Int J Innov Comp Inf Control 6(11), 5179-5190 (2010)

12. S Misra, PV Krishna, V Saritha, An efficient approach for distributed channel allocation with learning automata-based reservation in cellular networks. Simul Trans Soc Model Simul Int 88(10), 1166-1179 (2012)

13. CZ Zhao, LC Gan, Dynamic channel assignment for large-scale cellular networks using noisy chaotic neural network. IEEE Trans Neural Netw 22(2), 222-232 (2011)

14. PV Krishna, NChSN lyengar optimal channel allocation algorithm with efficient channel reservation for cellular networks. Int J Commun Netw Distrib Syst 1(1), 33-51 (2008)

15. S Bhattacharya, HM Gupta, S Kar, Performance modeling of cellular mobile systems: a review of recent advances. IETE Tech Rev 27(1), 15-26 (2010)

16. HS Kim, DH Kim, Ant colony optimization algorithm for centralized dynamic channel allocation in multi-cell OFDMA systems. IEICE Trans Commun E93B(6), 1475-1483 (2010)

17. SH Kim, YJ Suh, A distributed channel assignment protocol for rate separation in wireless mesh networks. Comput Commun 33(1), 1281-1295 (2010)

18. PV Krishna, S Misra, MS Obaidat, V Saritha, An efficient approach for distributed dynamic channel allocation with queues for real-time and non-real-time traffic in cellular networks. J Syst Softw 82(7), 1112-1124 (2009)

19. Gl Tsiropoulos, DG Stratogiannis, HH Chen, PG Cottis, Utility-based probabilistic call admission control for complete fairness in wireless networks. Int J Commun Syst 27(3), 521-528 (2014)

20. MZ Chowdhury, YM Jang, ZJ Haas, Call admission control based on adaptive bandwidth allocation for wireless networks. J Commun Netw 15(1), 15-24 (2013)
21. HT Lim, YH Kim, SH Pack, CH Kang. Call admission control with heterogeneous mobile stations in cellular/WLAN interworking systems. EURASIP J Wirel Comm. 2011; 91 (2011).

22. A Bozkurt, R Akdeniz, E Uçar. Call admission control jointly with resource reservation in cellular wireless networks. EURASIP J Wirel Comm. 2010; 740575 (2010).

23. M Ghaderi, R Boutaba, Call admission control in mobile cellular networks: a comprehensive survey. Wirel Commun Mob Comput 6(1), 69-93 (2006)

24. S Ghosh, A Konar, Call Admission Control in Mobile Cellular Networks (Springer, New York, 2012)

25. CX Li, SG Anavatti, T Ray, Analytical hierarchy process using fuzzy inference technique for real-time route guidance system. IEEE Trans Intell Transp Syst 15(1), 84-93 (2014)

26. K Nasrollahzadeh, MM Basini, Prediction of shear strength of FRP reinforced concrete beams using fuzzy inference system. Expert Syst Appl 41(4), 1006-1020 (2014)

27. GR Chen, TT Pham, Introduction to Fuzzy Systems (Chapman and Hall/CRC, Boca Raton, 2006)

\section{Submit your manuscript to a SpringerOpen ${ }^{\odot}$ journal and benefit from:}

- Convenient online submission

- Rigorous peer review

- Immediate publication on acceptance

- Open access: articles freely available online

- High visibility within the field

- Retaining the copyright to your article

Submit your next manuscript at $>$ springeropen.com 